\title{
ENTANGLEMENT PROPERTIES OF QUANTUM MANY-BODY WAVE FUNCTIONS
}

\author{
J. W. Clark, ${ }^{1}$ A. Mandilara, ${ }^{1}$ and M. L. Ristig ${ }^{2}$ \\ Department of Physics, Washington University \\ St. Louis, Missouri 63130, USA \\ ${ }^{2}$ Institut für Theoretische Physik, Universität zu Köln \\ D-50937 Köln, Germany
}

\section{INTRODUCTION}

The quantum information community is currently engaged in a major effort to quantify the entanglement content of states of multipartite quantum systems [1-9]. A multipartite system is made up of a number of parts, which may be identified with individual particles or with groups of particles. Entanglement (actually, Verschränkung is the name given by Schrödinger [10] to the nonlocal correlations responsible for violations of the Bell inequalities [11]. This property has emerged as a physical resource $[12,13]$, analogous to energy as a resource for useful work, which is to be drawn upon in schemes for quantum communication and quantum computation.

Since entanglement represents uniquely quantal correlations, it becomes of great interest to elucidate the entanglement properties of the wave functions commonly used to describe strongly correlated quantum many-body systems in condensedmatter physics, hadronic physics, and quantum chemistry. The information gained in such a program should improve our understanding of quantum phase transitions occurring in these systems as well as their behavior in regions away from critical points. Here we shall take a first step in this direction by quantifying the entanglement of correlated variational wave functions that have been developed to treat model systems of interacting Pauli spins localized on the sites of a regular lattice, i.e., spin-lattice models $[14,15]$.

A pure state of a multipartite quantum system is entangled if and only if its state vector is non-separable, meaning that it is not the direct product of state vectors of the parts. In many-body language, reading "parts" as "particles," the wave function cannot be written as a product of single-particle wave functions of some basis. A mixed state, which may generally be represented by a density operator, 
is nonseparable and therefore entangled if and only if it is not decomposable as a mixture of product states.

Exchange correlations in Fermi and Bose ground states do not contribute to entanglement as a useful resource [16-19]; accordingly, a state described by a single Slater determinant or permanent is to be considered separable. Thus, in examining the entanglement of a many-body wave function, one is in essence addressing its nonmean-field properties, which reflect fluctuation effects due to the presence of strong interactions. Any subset of the particles in a system of interacting particles in a pure state is necessarily in a mixed state.

Bipartite (two-party) entanglement of pure and mixed states has received thorough study, especially for the case that the two subsystems are two-level systems or Pauli spins. (This is of course the case of most immediate concern for quantum computation, where the two-level computing elements are called qubits.) While the quantification of bipartite entanglement is well under control, analysis of multipartite entanglement quickly becomes a formidable problem as the number of parties increases beyond three. For an $N$-partite quantum system, $N>2$, entanglement is not characterized by a single quantity, but rather by a non-unique set of quantities that grows polynomially with increasing $N$. Understandably, there is as yet no consensus on the best choice of such quantities.

In the present work we will consider only (i) bipartite entanglement of a single spin with the rest of the spins in the lattice and (ii) bipartite entanglement of two spins in the lattice environment. Accordingly, our treatment of spin-lattice models will involve the following standard measures of bipartite entanglement [20-22]: von Neumann entropy, entanglement of formation, concurrence, and localizable entanglement. Considering the transverse Ising model laid out on regular lattices (square, cube, tessaract) in two, three, and four dimensions, information on these quantities will be gathered from available results on the one- and two-site (or one- and twospin) density matrices corresponding to Hartree-Jastrow ground-state wave functions $[23-25,15]$. Where possible, comparison will be made with results of earlier work on exactly soluble models $[26,27,22]$ or stochastic simulation methods [28]. We also make an interesting simple connection of the Hartree-Jastrow functions with the nilpotent polynomial representation of entanglement [9], which permits us to expose important qualitative features of these trial ground states.

Section II provides the necessary formal and conceptual background on entanglement measures and their possible role in identifying and characterizing quantum phase transitions. In Section III we introduce the transverse Ising model and sketch its analysis and treatment within the framework of correlated-basis theory and hypernetted-chain techniques. The numerical results and attendant discussion are presented in Section IV. In Section V we look ahead to more ambitious explorations of the entanglement properties of the many-body wave functions employed in correlated-basis and coupled-cluster approaches to strongly correlated quantum systems. 


\section{ENTANGLEMENT AND QUANTUM PHASE TRANSITIONS}

The entanglement of the two parts $(1,2)$ of a bipartite system in a pure state $\rho=|\psi\rangle\langle\psi|$ may be defined as the von Neumann entropy

$$
S=-\operatorname{tr}\left(\rho_{i} \log _{2} \rho_{i}\right)=-\operatorname{tr}\left(\rho_{j} \log _{2} \rho_{j}\right)
$$

of either subsystem, where $\rho_{i}=\operatorname{tr}_{j}(\rho)$ and $\rho_{j}=\operatorname{tr}_{i}(\rho)$. When either subsystem is a spin-1/2 system, $S$ ranges from 0 (not entangled) to 1 (maximally entangled).

The entanglement between two parts of a system in a mixed state $\rho$ (e.g., two spins within a multispin system) is not uniquely defined. One natural definition is the entanglement of formation, which is the minimum, over all pure-state decompositions of $\rho$, of the expected entanglement required to construct $\rho$ from such a decomposition, using $S$ as a measure of the pure-state entanglement. In general, this quantity is awkward to calculate. However, for the case of two spins 1/2 (2 qubits), it can easily be found [21] from their density matrix as a simple monotonic function of the concurrence $C$,

$$
E_{F}(\rho)=h\left(\frac{1+\sqrt{1-C^{2}}}{2}\right)
$$

with

$$
h(x)=-x \log _{2} x-(1-x) \log _{2}(1-x) .
$$

Given the two-spin density matrix $\rho_{i j}$ obtained by tracing out all spins other than $i$ and $j$, the concurrence is calculated as

$$
C\left(\rho_{i j}\right)=\max \left[0, \mu_{1}-\mu_{2}-\mu_{3}-\mu_{4}\right],
$$

where the $\mu_{i}$ are the eigenvalues (in decreasing order, each real and nonnegative) of the Hermitian matrix

$$
R=\left[\rho^{1 / 2} \tilde{\rho} \rho^{1 / 2}\right]^{1 / 2}
$$

and

$$
\tilde{\rho}=\left(\sigma^{y} \otimes \sigma^{y}\right) \rho^{*}\left(\sigma^{y} \otimes \sigma^{y}\right)
$$

is the spin-flipped density matrix ( $\rho_{i j}$ being abbreviated as $\rho$ ). The concurrence $C$ ranges from zero for a separable state to unity for a maximally entangled state. For a pure state of qubits, $|\psi\rangle=a|00\rangle+b|01\rangle+c|10\rangle+d|11\rangle$, one obtains $C=|a d-b c|$, which is clearly a measure of the departure from a product state.

Other measures of bipartite entanglement of mixed states have also been proposed. The entanglement of assistance is the maximal two-party (e.g., two-spin) entanglement that can be achieved by performing any kind of measurement on the other parts of a multipartite (e.g., multispin) system. In a sense, this measure lies at the opposite extreme from the entanglement of formation, and again it is hard to calculate.

Verstraete et al. and Popp et al. [22] have proposed a similar measure that is more accessible. The localizable entanglement is the maximal amount of entanglement between two parties that can, on average, be created - localized - by performing 
only local measurements on the other parts of the system. Unlike the other bipartite entanglement measures mentioned, it is not (in general) determined from a knowledge of two-particle correlation functions alone. On the other hand, it captures more complicated features of the state of a multipartite system and leads to a natural definition of entanglement length. Moreover, although difficult to calculate in general, one can obtain bounds (usually tight ones) on its magnitude from the connected twoparty correlation function. An upper bound is given by the entanglement of assistance as measured by its concurrence $C_{A}$, while a lower bound is provided by the maximal connected (or "classical") correlation function (see Ref. [22] for details).

Our study of the entanglement properties of correlated wave functions for spin lattices was motivated by the work of Osterloh et al. [26] and more especially that of Osborne and Nielsen (ON) [27]. These authors were the first to explore possible connections between quantum phase transitions and entanglement. Both investigations focused on the anisotropic XY model on a one-dimensional (1D) lattice with $N$ sites occupied by Pauli spins with nearest-neighbor ferromagnetic couplings, subject to a transverse magnetic field. Since this model is exactly soluble using the JordanWigner transform, it admits an incisive analysis of the behavior of entanglement in the vicinity of a simple quantum phase transition from paramagnetic disorder to ferromagnetic order. A special case, the transverse Ising model, received the most attention. There was much subsequent work on quantum spin chains along similar lines, driven by their tractability and by the equivalence of spin- $1 / 2$ with the qubit of quantum information theory.

A quantum phase transition is associated with a qualitative change of the ground state of a quantum many-body system as some parameter (e.g, density, pressure, doping, coupling constant) is varied. In contrast to ordinary phase transitions driven by thermal fluctuations at finite temperature, quantum phase transitions are driven by purely quantal fluctuations and can occur at zero temperature. At the critical point in parameter space where the transition takes place, long-range correlations develop in the ground state. Osterloh et al. and ON proposed that there must exist an intimate relation between quantum phase transitions and entanglement, and that the behavior of a suitable entanglement measure should bear a signature of the singular behavior of the system near the critical point. Their results generally support this view, although some unexpected features were encountered. For example, the maximum of the nearest-neighbor concurrence does not occur exactly at the critical point, but at a slightly lower value of $\bar{\lambda}$. Importantly, these studies indicate that one cannot establish a universal connection between bipartite entanglement and quantum critical points, but rather that multipartite measures are necessarily involved in a rigorous analysis.

Let us consider the transverse Ising model in the form studied by ON:

$$
H=-\sum_{j=0}^{N-1}\left(\bar{\lambda} \sigma_{j}^{x} \sigma_{j+1}^{x}+\sigma_{j}^{z}\right) .
$$

In this form, the constants defining the model are lumped into a single coupling parameter $\bar{\lambda}$. ON examined the entanglement properties of both the ground state at zero temperature and the thermal mixed state at finite temperature $T$, observing that the ground state has a two-fold degeneracy which is generally broken. We shall be 
concerned only with their results for the ground state, whose bipartite entanglement content they measured in terms of (i) the von Neumann entropy $S$ of the one-site reduced density matrix and (ii) the concurrence between two spins, calculated from the two-site reduced density matrix. In the first case the two parties are a single spin $i \mathrm{j}$ and the $N-1$ spins making up the rest of the lattice system; in the second they are two spins $i j$, residing in a mixed state within the lattice system of the remaining $N-2$ spins.

Single-Site Entanglement. The one-site reduced density matrix for a spin $i$ at an arbitrary site (all being equivalent by translational invariance) is

$$
\rho_{i}=\operatorname{tr}_{i}(\rho)=\frac{1}{2} \sum_{\alpha=0}^{3} q_{\alpha} \sigma_{i}^{\alpha}
$$

where $\sigma^{0}=1$ and $\alpha=1,2,3$ are $x, y, z$, and

$$
q_{\alpha}=\operatorname{tr}\left(\sigma_{i}^{\alpha} \rho\right)=\left\langle\sigma_{i}^{\alpha}\right\rangle
$$

If the full symmetries of the Hamiltonian $H$ are enforced, the number of terms reduces to just one $(\alpha=1)$. However, the degeneracy of the ground state leads to broken symmetry with $q_{3} \neq 0$. The two parameters required to specify the single-site density matrix in the ground state are the longitudinal ( $x$ component) and transverse $(z$ component) magnetizations in either of the two degenerate states, say

$$
M_{x}=\left\langle 0^{+}\left|\sigma_{x}\right| 0^{+}\right\rangle, \quad M_{z}=\left\langle 0^{+}\left|\sigma_{z}\right| 0^{+}\right\rangle \text {. }
$$

Thus we have

$$
\rho_{i}=\frac{1}{2}\left(I+M_{x} \sigma_{x}+M_{z} \sigma_{z}\right)
$$

and the entanglement as given by von Neumann entropy is

$$
S \equiv-\operatorname{tr}\left(\rho_{i} \log _{2} \rho_{i}\right)
$$

with $0 \leq S \leq 1$. The two eigenvalues of $\rho_{i}$ are easily found to be

$$
\kappa_{1,2}=\frac{1}{2}\left(1 \pm \sqrt{M_{x}^{2}+M_{z}^{2}}\right)
$$

leading to the result

$$
\begin{aligned}
S & =-\kappa_{1} \log _{2} \kappa_{1}-\kappa_{2} \log _{2} \kappa_{2} \\
& =-\frac{1}{2}\left[(1+x) \log _{2}(1+x)+(1-x) \log _{2}(1-x)\right]
\end{aligned}
$$

for the von Neumann entropy of a single spin with respect to the rest of the lattice, where

$$
x^{2}=M_{x}^{2}+M_{z}^{2} .
$$


Two-Site Entanglement. For the two-site reduced density matrix, similar arguments lead to

$$
\rho_{i j}=\operatorname{tr}_{i j}(\rho)=\frac{1}{4} \sum_{\alpha, \beta=0}^{3} p_{\alpha \beta} \sigma_{i}^{\alpha} \otimes \sigma_{j}^{\beta},
$$

with coefficients

$$
p_{\alpha \beta}=\operatorname{tr}\left(\sigma_{i}^{\alpha} \sigma_{j}^{\beta} \rho\right)=\left\langle\sigma_{i}^{\alpha} \sigma_{j}^{\beta}\right\rangle,
$$

and to the expression

$$
\rho_{i j}=\frac{1}{4}\left[I+M_{z}\left(\sigma_{i}^{z}+\sigma_{j}^{z}\right)+\sum_{\alpha=1}^{3}\left\langle\sigma_{i}^{\alpha} \sigma_{j}^{\alpha}\right\rangle \sigma_{i}^{\alpha} \sigma_{j}^{\alpha}\right]
$$

in terms of the transverse magnetization $M_{z}$ and the two-spin correlation functions $\left\langle\sigma_{i}^{x} \sigma_{j}^{x}\right\rangle,\left\langle\sigma_{i}^{y} \sigma_{j}^{y}\right\rangle$, and $\left\langle\sigma_{i}^{z} \sigma_{j}^{z}\right\rangle$. Knowing these ingredients from an exact solution or approximate many-body treatment, the concurrence $C$ may then be determined from Eq. (4).

\section{VARIATIONAL THEORY OF TRANSVERSE ISING MODEL}

We now review the variational-CBF approach [23-25] to the ground state and elementary excitations of the transverse Ising model in $D$ dimensions. Here, "variational" means that a variational Ansatz is made for the ground-state wave function; "CBF" means "correlated basis functions," implying that both the ground-state and excited-state descriptors will contain nontrivial correlations beyond mean-field theory. Here we are only interested in the results for the magnetizations and correlation functions in the ground state required for the evaluation of the relevant bipartite entanglement measures.

Written for arbitrary dimension $D$, the Hamiltonian is written with a more general parametrization than that employed in Refs. [26,27,22], namely

$$
\mathcal{H}=\frac{1}{2} \sum_{i, j}^{N} \Delta_{i j} \sigma_{i}^{x} \sigma_{j}^{x}+\lambda \sum_{i}^{N}\left(1-\sigma_{i}^{z}\right) .
$$

The $N$ spins are situated on the lattice sites of a $D$-dimensional hypercube. A generic vector from one site to another will be denoted by $\mathbf{n}$. The spin-spin interaction is of the Ising type:

$$
\Delta(\mathbf{n})=\left\{\begin{array}{rl}
2 D & \mathbf{n}=0 \\
-1 & \text { for nearest neighbors } \\
0 & \text { otherwise }
\end{array}\right.
$$

with $\Delta_{i j} \equiv \Delta\left(\mathbf{r}_{i}-\mathbf{r}_{j}\right)=\Delta(\mathbf{n})$. The strength of the external field is measured by the coupling parameter $\lambda(0 \leq \lambda \leq \infty)$. For the special case $D=1$ (which we do not treat numerically), identification of $\lambda$ with $\bar{\lambda}$ brings the Hamiltonian (19)-(20) into coincidence with the form (7) used by Osborne and Nielsen, apart from an overall constant factor and a constant shift of energy. 
CBF theory provides a comprehensive framework for ab initio microscopic description of strongly interacting many-body systems [29]. In application to the transverse Ising model, one would like to achieve such a description for values of the coupling parameter $\lambda$ over its full range from 0 (corresponding to the strong-coupling limit) to $\infty$ (weak-coupling limit). Gross properties to be determined include the longitudinal magnetization $M_{x}=\left\langle\sigma_{i}^{x}\right\rangle$ in the normalized ground state, the transverse magnetization $M_{z}=\left\langle\sigma_{i}^{z}\right\rangle$, the spin-spin spatial distribution function $g(\mathbf{n})=\left\langle\sigma_{i}^{x} \sigma_{j}^{x}\right\rangle$, and the corresponding structure function $S(\mathbf{k})$, all in the ground state. Further, one would like to determine the ground-state energy $E_{0}$ and the coupling parameter $\lambda_{c}$ at the quantum critical point, where the system changes phase from paramagnetic to ferromagnetic (or vice versa). (In general one would also like to find the properties of the elementary excitations, including the dispersion law and magnon energies.)

To separate the mean-field effects from the effects of dynamical correlations, it is convenient to introduce a modified ("connected") distribution function

$$
G(\mathbf{n})=\left(1-M_{x}^{2}\right)^{-1}\left[g(\mathbf{n})-\delta_{\mathbf{n} \mathbf{0}}-\left(1-\delta_{\mathbf{n} \mathbf{0}}\right) M_{x}^{2}\right]
$$

and extract the so-called spin-exchange strength from $M_{z}$ :

$$
n_{12}=\left(1-M_{x}^{2}\right)^{-\frac{1}{2}} M_{z}
$$

In mean-field approximation, $G(\mathbf{n}) \equiv 0$ and $n_{12} \equiv 1$.

The following steps have been taken in the CBF analysis of the transverse Ising model, and corresponding numerical results are available [23-25]:

(i) Expression of the ground-state energy as a closed functional of the longitudinal magnetization $M_{x}$, the modified distribution function $G(\mathbf{n})$, and the spinexchange strength $n_{12}$.

(ii) Construction of a variational ground state having the essential correlation structure.

(iii) Evaluation of the spatial distribution function and spin-exchange strength, and hence the energy functional, for a generic trial ground state.

(iv) Optimization of the trial ground state - derivation and solution of Euler-Lagrange equations.

(v) Evaluation of the desired gross properties and correlation measures, for the optimal ground state.

(vi) Construction of the excited states and associated energies, in Feynman approximation.

Further steps have been envisioned but not carried out:

(vii) Systematic improvement of the zero-temperature description, by inclusion of higher-spin correlations and backflow in ground-state trial function and excitation Ansatz, and/or perturbation theory in a basis of correlated states.

(viii) Extension to finite $T$ via correlated density matrix theory [30].

For the purpose of the present work, only the first two steps, (i) and (ii), require more explicit presentation. The expression for the energy functional, applicable to a 
generic proposal for the ground state, is given by

$$
\frac{E_{0}}{N}\left[G(\mathbf{n}), M_{x} ; \lambda\right]=\left(1-M_{x}^{2}\right)\left[D+\frac{1}{2} \sum_{\mathbf{n}} \Delta(\mathbf{n}) G(\mathbf{n})\right]+\lambda\left[1-\left(1-M_{x}^{2}\right)^{\frac{1}{2}} n_{12}\right] .
$$

It turns out that the spin-exchange strength is dependent on $G$ and $M_{x}$. In the paramagnetic phase, the order parameter $M_{x}$ vanishes identically and $E_{0} / N$ becomes a functional only of $G$.

In mean-field theory, $G(\mathbf{n}) \equiv 0$ and $n_{12}=1$, so in this case $E_{0}$ becomes a function of $M_{x}$ only:

$$
\frac{E_{0}}{N}\left(M_{x}\right)=\left(1-M_{x}^{2}\right) D+\lambda\left[1-\left(1-M_{x}^{2}\right)^{\frac{1}{2}}\right]
$$

This function is minimized by $M_{x}=\left[1-(\lambda / 2 D)^{2}\right]^{\frac{1}{2}}$, implying a critical point at $\lambda_{c}=2 D$, beyond which $M_{x} \equiv 0$. The resultant optimal energy is given by $E_{0} / N=D$ in the disordered (paramagnetic) phase $(\lambda>2 D)$ and by $E_{0} / N=\lambda(1-\lambda / 4 D)$ in the ordered (ferromagnetic) phase $(0 \leq \lambda \leq 2 D)$.

Turning to the choice of variational wave function, much of the physics of the transverse Ising model can be captured by a correlated trial ground state of HartreeJastrow form:

$$
\left|\Psi_{\mathrm{HJ}}\right\rangle=\exp \left(M_{x} U_{M_{x}}+U\right)|0\rangle
$$

with

$$
\begin{gathered}
U=\frac{1}{2} \sum_{i<j}^{N} u\left(\mathbf{r}_{i j}\right) \sigma_{i}^{x} \sigma_{j}^{x}, \\
U_{M}=\sum_{i}^{N} u_{1}\left(\mathbf{r}_{i}\right) \sigma_{i}^{x}+\frac{1}{4} \sum_{i<j}^{N} u_{M}\left(\mathbf{r}_{i j}\right)\left(\sigma_{i}^{x}+\sigma_{j}^{x}\right) .
\end{gathered}
$$

The vacuum or reference state $|0\rangle$ is taken as a symmetric product of $N$ single-spin states with spin components $\sigma_{i}^{z}=+1$, i.e.

$$
|0\rangle=|\uparrow \uparrow \cdots \uparrow\rangle_{N}
$$

For a translationally invariant system, the single-spin function $u_{1}\left(\mathbf{r}_{i}\right)$ is independent of the lattice site $\mathbf{r}_{i}$, while the two-body pseudopotentials $u$ and $u_{M}$ depend only on the relative distance $\mathbf{n}$, where $\mathbf{n}=\mathbf{r}_{i j}=\mathbf{r}_{i}-\mathbf{r}_{j}$. In mean-field approximation, $u(\mathbf{n}) \equiv u_{M}(\mathbf{n}) \equiv 0$.

In the disordered phase, the $U_{M}$ generator is not present in the exponential form defining the correlated trial ground state, since the order parameter $M_{x}$ vanishes identically. However, this term makes a vital contribution in the ordered phase, where it is responsible for the symmetry breaking. (Note that reflection in a mirror plane normal to the $x$-axis transforms $U_{M}$ to $-U_{M}$ and reveals a two-fold degeneracy of the ordered ground state (characterized by the magnetizations $M_{x}$ and $-M_{x}$ ). It should also be mentioned that the pseudopotential $u_{M}(\mathbf{n})$ is in fact a functional of the generator $u(\mathbf{n})$. 
Evaluation of the energy functional $E_{0}\left[G\left(\mathbf{n}, M_{x} ; \lambda\right)\right]$ requires construction of the spatial distribution function $G(\mathbf{n})$ and the spin-exchange strength $n_{12}$ corresponding to the trial ground state, as functionals of the pseudopotential $u(\mathbf{n})$ that generates the spatial correlations. This is done efficiently by exploiting a $1-1$ mapping of the spin-lattice system onto a binary mixture of two boson species, made possible by the assumed form of the trial ground state. The two boson species are characterized by eigenvalues +1 and -1 of the spin operator $\sigma^{x}$ and may be called particles and holes, respectively. The partial densities $\rho_{+}$and $\rho_{-}$of particle and hole components are determined by the magnetization $M_{x}$ through $\rho_{ \pm}=\frac{1}{2}\left(1 \pm M_{x}\right)$, i.e., by the expectation values $\rho_{ \pm}=\left\langle P_{i}^{( \pm)}\right\rangle$of the projectors $P_{i}^{( \pm)}=\frac{1}{2}\left(1 \pm \sigma_{i}^{x}\right)$. The Hypernetted-Chain (HNC) analysis available for the Hartree-Jastrow ground state of the binary boson mixture [31] may then be applied to determine all the requisite quantities for the corresponding variational description of the transverse Ising system.

The trial ground state is optimized by deriving and solving suitable EulerLagrange equations that determine the optimal distribution function $G(\mathbf{n})$ the magnetization $M_{x}$. For constant $\lambda$ and $M_{x}$, the optimal $G(\mathbf{n})$ is determined, through its HNC connection to the pseudopotential $u(\mathbf{n})$, by means of the Euler-Lagrange equation $\delta E_{0} / \delta u(\mathbf{n})=0$ for $u(\mathbf{n})$, which leads to a paired-magnon equation [24]. Similarly, variation of the energy functional with respect to $M_{x}^{2}$ at constant $\lambda$ and fixed $G(\mathbf{n})$ produces an Euler-Lagrange $\partial E_{0} / \partial M^{2}=0$ equation for the optimal order parameter in the ordered phase (moot in the disordered phase), which leads to a renormalized Hartree equation [24].

This approach yields exact results in strong and weak-coupling limits, and good results in between, but it cannot be expected to reproduce critical exponents without the inclusion of higher-spin correlations.

\section{ENTANGLEMENT IN HARTREE-JASTROW GROUND STATES}

Numerical data are available from published [23-25] and unpublished variational$\mathrm{CBF}$ calculations in the transverse Ising model that suffice for meaningful evaluations of measures of corresponding bipartite entanglement properties for two-, three-, and four-dimensional $(D=2,3,4)$ versions of the model.

Single-Site Entropy. Eqs. (14) and (15) are used to quantify the entanglement of a single site with the rest of the lattice (the single-spin von Neumann entropy defined in Section II), using the variational-CBF inputs for $M_{x}$ and $M_{z}$ for many choices of the coupling parameter $\lambda$. The results, plotted in Fig. 1, indicate a sharp peaking of $S(\lambda)$ at the critical values of $\lambda$ for the order-disorder transition from ferromagnetism to paramagnetism given by the many-body calculation (respectively, $\lambda_{c}=3.14,5.12$, and 7.1 for $D=2,3$, and 4 ). It is tempting to interpret this peaking, with the entanglement measure reaching a maximum at the transition, in terms of a direct association of entanglement with quantum critical phenomena. The same behavior was observed by Osborne and Nielsen for $D=1$, however with a distinctly higher maximum value of $S$ (0.68 in comparison with the value 0.22 we find at $D=2$ ). In fact, the maxima are seen to decline systematically as $D$ increases. This finding is in harmony with the general understanding that classicality increases with dimension. 


\section{Transverse Ising Model}

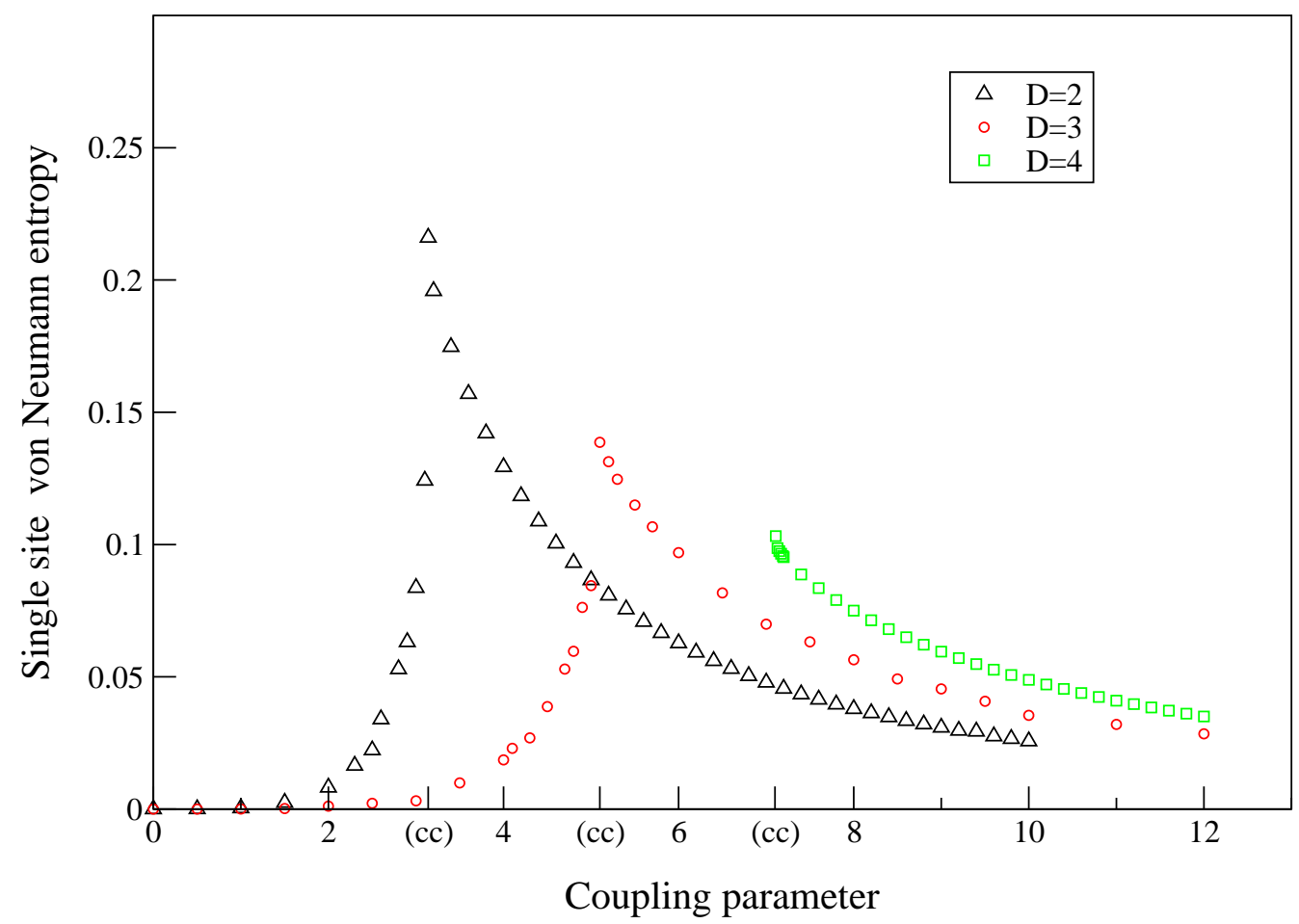

Figure 1. Von Neumann entropy $S$ between a single site and the remaining sites in a square, cubic, or hypercubic lattice (respectively for dimensions $D=2,3$, or 4 ), evaluated with input data from variational-CBF calculations based on optimized Hartree-Jastrow ground-state trial functions. The locations of the respective critical coupling parameters $\lambda_{c}$ are labeled with (cc).

This suggests that in practical implementations of quantum information processing where entanglement is used as a resource, it is advantageous to utilize chains of processing units rather than arrays in higher dimensions.

Two-Site Entanglement. We next study the entanglement between two spins residing in the lattice in terms of the concurrence defined in Section II, deriving information on these measures from the data available from the CBF-variational studies based on the optimized Hartree-Jastrow trial function. In applying Eq. (4), We may use the following formulas for the eigenvalues $\mu_{i}$ of the matrix $R$ of Eq. (5) in terms of spin-spin correlation functions, which are valid in both ordered and disordered phases

$$
\begin{gathered}
\mu_{1,2}=\frac{1}{4}\left|1-\left\langle\sigma_{i}^{z} \sigma_{j}^{z}\right\rangle \pm\left(\left\langle\sigma_{i}^{x} \sigma_{j}^{x}\right\rangle+\left\langle\sigma_{i}^{y} \sigma_{j}^{y}\right\rangle\right)\right| \\
\mu_{3,4}=\frac{1}{4}\left|\left[\left(1+\left\langle\sigma_{i}^{z} \sigma_{j}^{z}\right\rangle\right)^{2}-\left\langle\sigma_{i}^{z}+\sigma_{j}^{z}\right\rangle^{2}\right]^{1 / 2} \pm\left(\left\langle\sigma_{i}^{x} \sigma_{j}^{x}\right\rangle-\left\langle\sigma_{i}^{y} \sigma_{j}^{y}\right\rangle\right)\right| .
\end{gathered}
$$




\section{Transverse Ising Model $(\mathrm{D}=2)$}

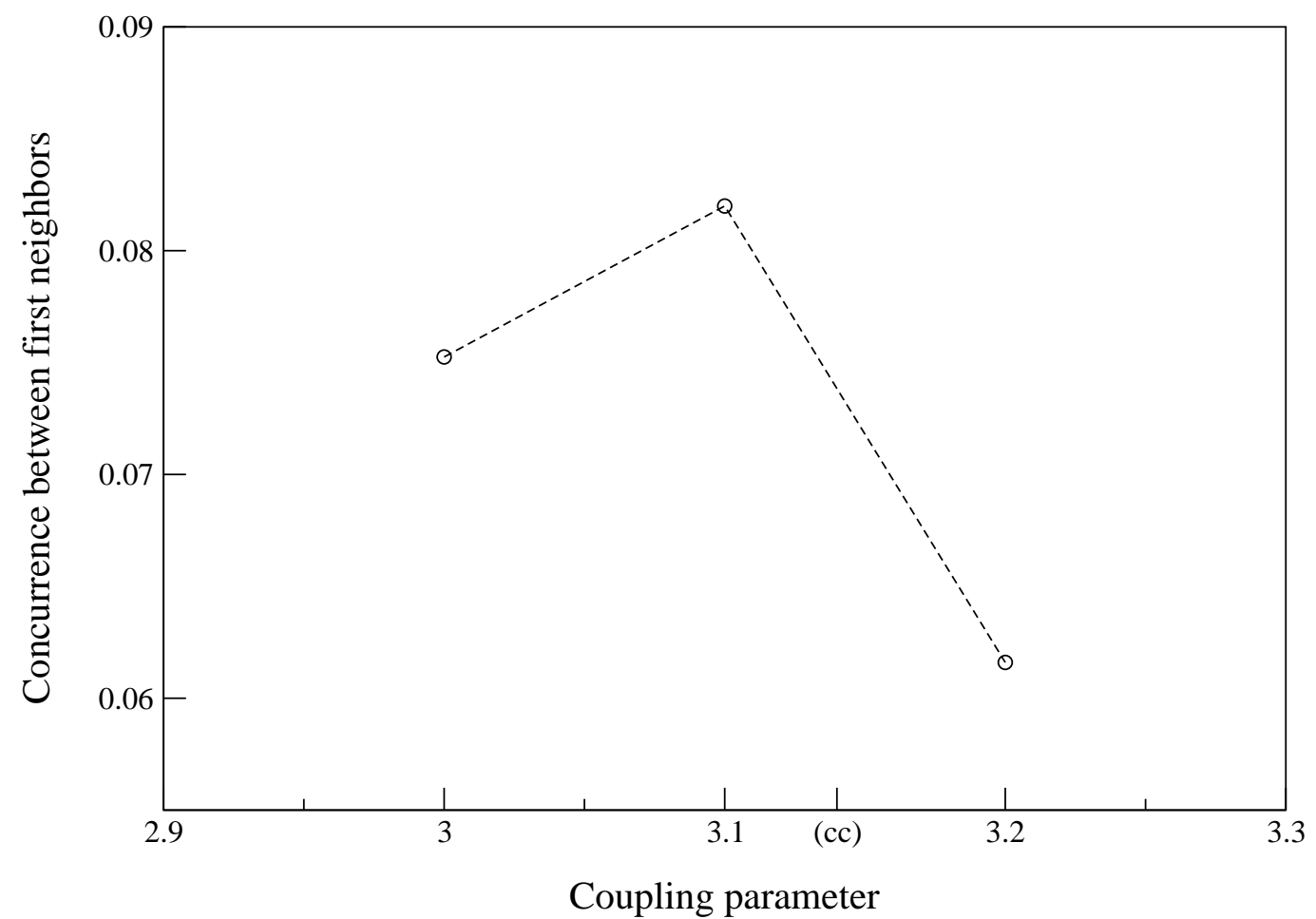

Figure 2. Concurrence $C$ between nearest neighbors in a square lattice, evaluated with input data from variational-CBF calculations based on an optimized HartreeJastrow ground-state trial function. The location of the critical coupling parameter $\lambda_{c}$ is labeled with (cc). The dashed line serves merely to guide the eye.

Concurrence results for nearest-neighbor spins in the two-dimensional model are plotted in Fig. 2 at three values of the coupling parameter $\lambda$. Since the existing data on the correlation functions is quite limited, this figure is not very informative. Still, it is of interest to point out that the values obtained are lower than those obtained by Syljuåsen [28] based on Monte Carlo simulation at finite temperatures, but the qualitative features are the same as those found in that work and by Osborne and Nielsen [27] and Osterloh et al. [26]. In our case, the peak may be closer to the critical $\lambda$ than is the case for the chain [27]. As noted in Ref. [28], one should expect the nearest-neighbor concurrence to be smaller in higher dimension due to the monogamous character of entanglement - the more neighbors, the smaller the share of bipartite entanglement allotted to each pair. Also, we know quite explicitly from the results of ON and Osterloh et al. that although spin-spin correlations in the usual sense acquire a long-range character upon approach to the critical point, this is not the case for the concurrence. In particular, these authors find that $C$ vanishes for site separations on the chain beyond next-nearest neighbor. In our case, since the scale of $C$ is already smaller because of the higher dimensionalities considered, the concurrence is found to vanish for pair separations beyond nearest neighbor. 


\section{Transverse Ising Model}

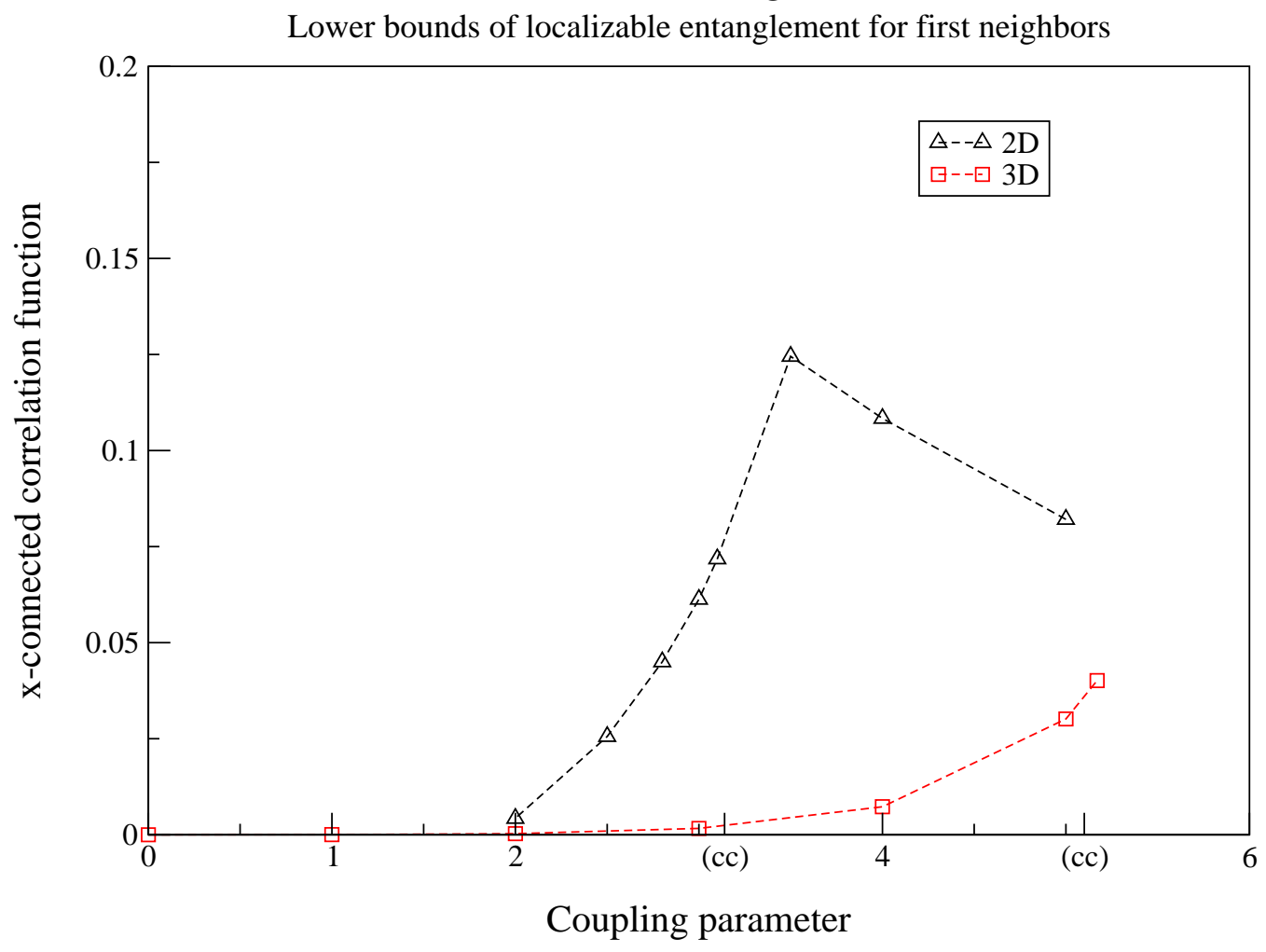

Figure 3. Two-point connected correlation function $Q_{x x}(|\mathbf{n}|)$ for nearest neighbors on a square or cubic lattice (respectively for dimensions $D=2$ or 3 ), evaluated with input data from variational-CBF calculations based on Hartree-Jastrow ground-state trial functions. The locations of the respective critical coupling parameters $\lambda_{c}$ are labeled with (cc). The dashed line serves merely to guide the eye.

Localizable Entanglement. As indicated in Section II, localizable entanglement is another recently suggested measure of bipartite entanglement [22]. It has the virtue of providing for a more natural definition and incisive definition of entanglement length than the entanglement of formation, and allowing one to observe its expected divergence at the quantum critical point. For the case of the transverse Ising model, the $x$-connected spin-spin correlation function $Q_{x x}(\mathbf{n})$, furnishes a tight lower bound on the localized entanglement, at least when nearest-neighbor spins are considered $[22]$.

Based on the theoretical analyses and numerical data of the variational-CBF work reported in Refs. [23-35], we have evaluated $Q_{x x}(|\mathbf{n}|)=g(|\mathbf{n}|)-M_{x}^{2}$ for a number of $\lambda$ values, in the cases $D=2$ and 3 (see Fig. 3). (Here, $|\mathbf{n}|$ denotes the spin-spin distance in the lattice at constant transverse magnetic field.) It is important to add that the data used are not fully optimal. If we compare with the corresponding results of Verstraete et al. [22], it is seen that the variational results for $Q_{x x}$ behave in the same way as when, in their treatment, a small perturbing magnetic field is imposed in the $x$ direction to break the parity symmetry (i.e., under $x \rightarrow-x$ ) and achieve a more realistic description in the strong-coupling limit (small $\lambda$ ). The 
treatment on which our input data is based already incorporates parity breaking in the ferromagnetic phase.

Tanglemeter. There have been some initial efforts toward analysis of multipartite entanglement in quantum spin models, focusing on spin chains [32-36]. In this vein, but not restricted to one dimension, we shall make some observations on the entanglement properties of Hartree-Jastrow ground states within the recently developed multipartite formalism based on nilpotent polynomials [9].

Given the ground state Eqs. (25)-(27) determined by the pseudopotentials $U$ and $U_{M}$, one can easily find the corresponding nilpotent polynomial $F$ defined in Ref. [9]. For the present argument it is irrelevant whether or not $U$ and $U_{M}$ are optimized. It is very important that $U$ and $U_{M}$ are respectively of two-body and one-body structure. Thus, the local contributions from $U_{M}$ can be ignored, since they do not affect the entanglement properties of the state. The required construction leading to the so-called tanglemeter is further simplified by the fact that the different terms and factors in $U$ commute with one another. The polynomial $F$ is then found to be

$$
F=1+\sum_{i<j} \alpha_{i j} \sigma_{i}^{+} \sigma_{j}^{+}+\sum_{i<j<k<l} \alpha_{i j k l} \sigma_{i}^{+} \sigma_{j}^{+} \sigma_{k}^{+} \sigma_{l}^{+}+\ldots+\alpha_{12 \ldots N} \sigma_{1}^{+} \sigma_{2}^{+} \ldots \sigma_{N}^{+}
$$

which does not contain terms linear or of order $N-1$ in the $\sigma^{+}$operators. Therefore $F$ is already in its canonical form $F_{c}$ under general local transformations, and the number of the coefficients is less than the minimum number of parameters sufficient to describe the entanglement properties of the state. The last step is to determine the logarithm of the polynomial $F$, the so-called nilpotential $f$. The result for $f$ is of the same form as $F$ and so already in canonical and hence tanglemeter.

From this simple analysis, we can infer that for any non-zero choice for the pseudopotential $U$ in the form (26), the ground state (25) belongs to the general orbit of states which contain $N$-partite entanglement. However, this state cannot be the GHZ state containing maximal $N$-partite entanglement. Another implication is that there are no subclusters of spins that are not entangled with the rest of the lattice. All spins share quantum correlations with all others.

\section{ENTANGLEMENT IN OTHER MANY-BODY WAVE FUNCTIONS}

Although much can still be learned from studies of entanglement in lattice spin systems, the characterization of this fascinating nonlocal quantal correlation in systems of particles having continuous spatial degrees of freedom as well as discrete spin/isospin observables presents the greatest opportunities for new insights relevant to strongly interacting quantum systems.

A typical CBF state has the form of a correlation factor applied to a reference independent-particle model state. The example of the transverse Ising model shows that a Jastrow factor introduces nontrivial entanglement properties. It is instructive to compare the simple Jastrow ground state for a normal Fermi system, namely $\prod_{i<j} f(i j) \Phi$ where $\Phi$ is a Slater determinant, with the BCS ground state projected on the subspace with the same even number $N$ of particles, which has the form $\mathcal{A}(\chi(12) \chi(34) \cdots \chi(N-1, N))$ (antisymmetrized by $\mathcal{A})$. The BCS wave function 
is evidently separable, being a mean-field description of Cooper pairs all in the twobody state $\chi$. Thus the useful entanglement is concentrated entirely in the individual Cooper pairs, which are not entangled one with the other. As demonstrated by Shi [19], the entanglement of each member of a Cooper pair with the other is given by a von Neumann entropy

$$
S=\left|v_{k}\right|^{2} \log \left|v_{k}\right|^{2}-\left(1-\left|v_{k}\right|^{2}\right) \log \left(1-\left|v_{k}\right|^{2}\right),
$$

where $v_{k}$ is the amplitude appearing in the usual expression

$$
|\mathrm{BCS}\rangle=\prod_{k}\left(u_{k}+v_{k} a_{k \uparrow}^{\dagger} a_{-k \downarrow}^{\dagger}\right)|0\rangle
$$

for the BCS state vector, with $\left|v_{k}\right|^{2}=(1 / 2)\left(1-\epsilon_{k} / E_{k}\right)$ and $E_{k}=\left(\epsilon_{k}+\Delta_{k}^{2}\right)^{1 / 2}$ in the usual notation. The pair-member entanglement is zero when the gap $\Delta_{k}$ vanishes (normal state), and the Cooper pairs are maximally entangled when $E_{k}>>\epsilon_{k}$.

By contrast, within the Jastrow product of pair functions $f$, entanglement is spread among all $N(N-1) / 2$ pairs $i j$, both directly and indirectly. Inclusion of interparticle correlations of all orders (between pairs, triples, quartets, etc.) is accomplished with the Feenberg function $\exp \left[\sum_{i<j} u_{2}(i j) / 2+\sum_{i<j<k} u_{3}(i j k) / 2+\cdots\right]$, which in itself can provide an exact representation of the ground state of a system of indistinguishable bosons [37]. A study of the multipartite entanglement properties of this function should prove very informative. (Such an analysis may be naturally extended to shadow wave functions [38], a generalization of the Jastrow-Feenberg form used predominantly in the study of quantum fluids and solids.)

For similar reasons, the exponential structure of the states of coupled-cluster theory [39],

$$
\left|\Psi_{\mathrm{CC}}\right\rangle=\exp \left[S_{1}+S_{2}+\cdots S_{N}\right]
$$

wherein

$$
S_{1}=\sum_{i j} c_{i j} a_{i}^{\dagger} a_{j}, \quad S_{2}=\sum_{i j k l} c_{i j k l} a_{i}^{\dagger} a_{j}^{\dagger} a_{k} a_{l}, \text { etc. }
$$

is a propitious format for the investigation of multipartite entanglement in manybody problems in nuclear physics and related areas, already begun by Emary [40]. Moreover, the CC structure suggests that a fruitful analysis can be performed in terms of the "tanglemeter" developed by Mandilara et al. [9] as an extensive characterization of entanglement based on nilpotent polynomials.

\section{ACKNOWLEDGMENTS}

The research described herein was supported in part by the U.S. National Science Foundation under Grant No. PHY-0140316. JWC is grateful to the US Army Research Office-Research Triangle Park for travel support through a grant to Southern Illinois University-Carbondale, which permitted him to take part in CMT29 at Kizu. He expresses sincere appreciation to the Japanese hosts of the the Workshop for their splendid organization, generosity, and hospitality. JWC and AM would also 
like to acknowledge partial support from FCT POCTI, FEDER in Portugal and the hospitality of the Centro de Cin̂cias Mathemáticas of the University of Madeira in connection with Madeira Math Encounters XXIX, during which much of this work was done.

\section{REFERENCES}

[1] V. Vedral, M. B. Plenio, K. Jacobs, and P. L. Knight, Phys. Rev. A 56, 4452 (1997); V. Vedral, B. Plenio, M. A. Rippin, and P. L. Knight, Phys. Rev. Lett. 78, 2275 (1998).

[2] N. Linden, S. Popescu, and A. Sudbery, Phys. Rev. Lett. 83, 243 (1999); H. A. Carteret, N. Linden, S. Popescu, and A. Sudbery, Found. Phys. 29, 527 (1999).

[3] W. Dür, G. Vidal, and J. I. Cirac, Phys. Rev. A 62, 062314 (2000).

[4] V. Vidral, Rev. Mod. Phys. 74, 197 (2002).

[5] J. Eisert, K. Audenaert, and M. B. Plenio, J. Phys. A: Math. Gen. 36, 5605 (2003).

[6] A. Miyake, Phys. Rev. A 67, 012108 (2003).

[7] F. Verstraete, J. Dehaene, and B. De Moor, Phys. Rev. A 68, 012103 (2003).

[8] H. Barnum, E. Knill, G. Ortiz, R. Somma, and L. Viola, Phys. Rev. Lett. 92, 107902 (2004); R. Somma, G. Ortiz, H. Barnum, E. Knill, and L. Viola, Phys. Rev. A 70, 042311 (2004).

[9] A. Mandilara, V. M. Akulin, A. V. Smilga, and L. Viola, Description of quantum entanglement with nilpotent polynomials, quant-ph/0508234 (2005), to be submitted to Phys. Rev. A.

[10] E. Schrödinger, Proceedings of the Cambridge Philosophical Society 31, 555 (1935).

[11] J. S. Bell, Rev. Mod. Phys. 38, 447 (1966).

[12] M. A. Nielsen and I. L. Chuang, Quantum Computation and Quantum Information (Cambridge University Press, Cambridge, 2000).

[13] J. Preskill, Lecture Notes on Quantum Computation, http://www.theory.caltech. $\mathrm{edu} / \mathrm{people} / \mathrm{preskill} / \mathrm{ph} 229 /$ \#Lecture.

[14] J. W. Clark and M. L. Ristig, Theory of Spin Lattices and Lattice Gauge Models, Springer Lecture Notes in Physics, Vol. 494 (Springer, Berlin, 1997).

[15] R. F. Bishop, D. J. J. Farnell, M. L. Ristig, Int. J. Mod. Phys. 14, 1517 (2000).

[16] J. Schliemann, D. Loss, and A. H. McDonald, Phys. Rev. B 63, 085311 (2001); J. Schliemann, J. I. Cirac, M. Kuś, M. Lewenstein, and D. Loss, Phys. Rev. A 64, 022303 (2001); K. Eckert, J. Schliemann, D. Bruss, and M. Lewenstein, Ann. Phys. (N.Y.) 299, 88 (2002).

[17] R. Paskauskas and L. You, Phys. Rev. B 64, 042310 (2001).

[18] Y. S. Li, B. Zeng, X. S. Liu, and G. L. Long, Phys. Rev. A 64, 054302 (2001).

[19] Y. Shi, J. Phys. A: Math. Gen. 37, 6807 (2004).

[20] C. H. Bennett, H. J. Bernstein, S. Popescu, and B. Schumacher, Phys. Rev. A 
53, 2046 (1996).

[21] W. K. Wootters, Quantum Inf. Comput. 1, 27 (2001); S. Hill and W. K. Wootters, Phys. Rev. Lett. 78, 5022 (1997); W. K. Wootters, Phys. Rev. Lett. 80, 2245 (1998).

[22] F. Verstraete, M. Popp, and J. I. Cirac, Phys. Rev. Lett. 92, 027901 (2004); M. Popp, F. Verstraete, M. A. Martín-Delagado, and J. I. Cirac, Phys. Rev. A 71, 042306 (2005). 032110 (2002).

[23] M. L. Ristig and J. W. Kim, Phys. Rev. B 53, 6665 (1996).

[24] M. L. Ristig, J. W. Kim, and J. W. Clark, in Theory of Spin Lattices and Lattice Gauge Models (Springer-Verlag, Berlin, 1997), p. 62.

[25] J. W. Kim, M. L. Ristig, and J. W. Clark, Phys. Rev. B 57, 56 (1998).

[26] A. Osterloh, L. Amico, G. Falci, and R. Fazio, Nature 416, 608 (2002).

[27] T. J. Osborne and M. A. Nielsen, Phys. Rev. A 66,

[28] O. F. Syljuåsen, Phys. Lett. A322, 25 (2004).

[29] J. W. Clark and E. Feenberg, Phys. Rev. 113, 388 (1959); J. W. Clark and P. Westhaus, Phys. Rev. 141, 833 (1966); E. Feenberg, Theory of Quantum Fluids (Academic Press, New York, 1969); C. E. Campbell and E. Feenberg, Phys. Rev. 188, 396 (1969); J. W. Clark, in The Many-Body Problem, Jastrow Correlations versus Brueckner Theory, Springer Lecture Notes in Physics, Vol. 138, R. Guardiola and J. Ros, eds. (Springer-Verlag, Berlin), p. 184; C. E. Campbell, in Recent Progress in Many-Body Theories, Vol. 4 E. Schachinger, H. Mitter, and H. Sormann, eds. (Plenum, New York, 1995), p. 29; E. Krotscheck, in Lecture Notes in Physics, Vol. 510, J. Navarro and A. Polls, eds. (Springer, Heidelberg, 1998).

[30] C. E. Campbell, K. E. Kürten, M. L. Ristig, and G. Senger, Phys. Rev. B 30, 3728 (1984); G. Senger, M. L. Ristig, K. E. Kürten, and C. E. Campbell, Phys. Rev. B 33, 7562 (1986); K. A. Gernoth, J. W. Clark, and M. L. Ristig, Z. Physik B 98, 337 (1995).

[31] M. L. Ristig, S. Fantoni, and K. E. Kürten, Z. Phys. B 51, 1 (1983).

[32] X. Wang, Phys. Rev. A 66, 044305 (2002).

[33] P. Stelmachovic and V. Buzek, Phys. Rev. A 70, 032313 (2004).

[34] A. Lakshminarayan and V. Subrahmanyam, quant-ph/0409039 (2005)

[35] D. Bruss, N. Datta, A. Ekert, L. C. Kwek, and C. Macchiavello, quant-ph/0411080 (2005).

[36] O. Guhne, G. Toth, and H. J. Briegel, quantph/0502160 (2005).

[37] J. W. Clark, Nucl. Phys. A328, 587 (1979).

[38] L. Reatto and G. L. Masserini, Phys. Rev. B 38, 4516 (1988).

[39] F. Coester, Nucl. Phys. 7, 421 (1958); F. Coester and H. Kümmel, ibid. 17, 477 (1960); J. Cizek, J. Chem. Phys. 45, 4256 (1966); Adv. Chem. Phys. 14, 35 (1969); R. F. Bishop and K. H. Lührmann, Phys. Rev. B 17, 3757 (1978); H. Kümmel, K. H. Lührmann, and J. G. Zabolitzky, Phys. Rep. 36C, 1 (1978); J. S. Arponen, Ann. Phys. (N.Y.) 151, 311 (1983); R. F. Bishop and H. Kümmel, Phys. Today 40(3), 52 (1987); J. S. Arponen, R. F. Bishop, and 
E. Pajanne, Phys. Rev. A 36, 2519 (1987); ibid. 36, 2539 (1987); R. J. Bartlett, J. Phys. Chem. 93, 1697 (1989); R. F. Bishop, Theor. Chim. Acta 80, 95 (1991).

[40] C. Emary, invited talk presented at the Conference on Microscopic Approaches to Many-Body Theory, University of Manchester, Aug 31-Sept 3, 2005; and private communication. 